Rabaska

Revue d'ethnologie de l'Amérique française

\title{
Le « boutonné » de Charlevoix : pertinence d'une découverte
}

\section{Anne-Marie Poulin}

Volume 13, 2015

Présence de Marius Barbeau : l'invention du terrain en Amérique

française. Autour d'un legs centenaire (1914-2014)

URI : https://id.erudit.org/iderudit/1033756ar

DOI : https://doi.org/10.7202/1033756ar

Aller au sommaire du numéro

Éditeur(s)

Société québécoise d'ethnologie

ISSN

1703-7433 (imprimé)

1916-7350 (numérique)

Découvrir la revue

Citer cet article

Poulin, A.-M. (2015). Le « boutonné » de Charlevoix : pertinence d’une découverte. Rabaska, 13, 131-140. https://doi.org/10.7202/1033756ar d'utilisation que vous pouvez consulter en ligne.

https://apropos.erudit.org/fr/usagers/politique-dutilisation/ 


\title{
Le «boutonné » de Charlevoix : pertinence d'une découverte
}

\author{
AnNe-Marie Poulin \\ Ethnologue-conseil, Québec
}

Il est heureux que l'énoncé de la problématique de ce colloque international, portant pour l'essentiel sur l'oralité, ait considéré l'apport de Marius Barbeau envers "d'autres composantes de la tradition ». Le présent texte évoque une de ces composantes pour examiner sa pertinence aux plans historique, esthétique et ethnologique.

Dans ses " Mémoires », toujours inédits, Marius Barbeau note avec enthousiasme, le résultat d'une visite à l'île aux Coudres en 1925, où il découvre enfin « cette fameuse boutonnée pour laquelle, écrit-il, je me serais rendu au bout du monde afin de l'obtenir et la sauvegarder. J'en fis l'acquisition comme de toutes celles jusqu'à quinze ou vingt ${ }^{1} »$. À l'évidence, ce pionnier qui avait déjà donné ses lettres de noblesse scientifique au folklore était bien renseigné et avait préparé son terrain. Cependant à l'époque, l'intérêt pour les traditions orales l'emporte sur la « découverte » d'un bien matériel. Aussi, a-t-elle peu d'impact sur la communauté scientifique, du moins dans l'immédiat. Par contre, sur la région et sur la technique qu'il visait tant à sauvegarder, l'impact est quasi instantané.

Précisons que la « fameuse boutonnée » tant recherchée par Barbeau est un couvre-lit tissé et que le mot boutonné est un régionalisme québécois. Polysémique, le terme qualifie aussi bien l'objet issu de la technique du tissage que le procédé. Le terme précis étant bouclé par la trame. En hommage au « découvreur» du boutonné, à la thématique du colloque ainsi que la région de la découverte, j'utilise le régionalisme qui est toujours en vigueur ici comme ailleurs au Québec, voire au-delà de ses frontières² ${ }^{2}$ Par définition, le boutonné

1. «Les Mémoires de Marius Barbeau », fonds Carmen Roy (2006-F0003) boîte 624, f.13, p.710. Je suis redevable à Benoît Thériault, archiviste au Musée canadien de l'histoire, de m'avoir signalé ce document.

2. Même chez le tisserand anglophone, le terme «boutonné » est reconnu et est écrit comme tel, tout comme celui de la technique du tissage « cannelé » d'Oscar Bériau, premier directeur de l'École des arts domestiques à Québec (1930). En anglais, les termes officiels sont tufted weave ou weft-loop weave. 
est une technique qui produit des motifs en relief en tissant. Historiquement, l'œuvre classique est généralement blanche et se pare de petites bouclettes ou boutons de coton blanc soulevés uniquement à l'aide des doigts en cours de tissage. Bien que Marius Barbeau ait souhaité que le boutonné origine de la région, la technique (tout comme plusieurs des contes qu'il a recueillis) est à la fois millénaire et universelle ${ }^{3}$. Plus près de notre époque, au $\mathrm{XVIII}^{\mathrm{e}}$ siècle, l'Espagne confectionne des couvre-lits et objets décoratifs avec des motifs similaires à ceux retrouvés en Charlevoix au siècle suivant (par exemple : l'étoile à huit branches ${ }^{4}$ ). Au début du XIX siècle, en Angleterre, une petite industrie locale à Bolton au Lancashire produit une couverture blanche boutonnée qui a pu inspirer des artisanes d'ici', tout comme celle produite peu de temps après, en Nouvelle-Angleterre à la Rutgers Factory à Paterson, New-Jersey. Ce modèle de boutonné classique et monochrome est tissé principalement sur la rive sud du Saint-Laurent, incluant la Beauce, Bellechasse, Kamouraska et Témiscouata, en passant par la Gaspésie et les provinces maritimes et vers l'ouest, en Estrie et en Ontario. L'influence des modèles de couvertures anglaises et anglo-américaines ne semble pas étrangère à cette incursion en sol québécois non plus, étant donné les échanges avec les exilés québécois travaillant dans les manufactures de textiles de la Nouvelle-Angleterre.

Dans Charlevoix, «pays à part » selon Barbeau ${ }^{6}$, la réalité est tout autre. De fait, les premières œuvres repérées au milieu du XIX ${ }^{\mathrm{e}}$ siècle montrent qu'elles diffèrent sensiblement du modèle classique évoqué. Le traditionnel boutonné charlevoisien se distingue par des couleurs vives, des bouclettes en laine teinte et une grande diversité de motifs ${ }^{7}$. Parmi eux, l'étoile à huit branches que l'on viendra à identifier, à tort, à la région de Charlevoix ${ }^{8}$. Nul recours ici aux canons de l'art savant et peu de recherche dans les proportions ou la symétrie, le but premier étant de produire la beauté sur un objet utilitaire

3. Michel Laurent, « Bouclé par la trame (dit boutonné)». La technique remonte à l'Antiquité et à la civilisation babylonienne et poursuit sa diffusion autour du bassin méditérranéen au cours des siècles pour rejoindre l'Europe et les pays nordiques. Le procédé est également pratiqué ailleurs comme au Pérou. Cf. Boutonné d'hier et d'aujourd'hui, Les Tisserands-Créateurs en collaboration avec Michel Laurent, Québec,1983, p. 9.

4. L'étoile à huit branches symbolise le soleil rayonnant. Tout comme la technique, les plus anciennes étoiles attestées proviennent de la Mésopotamie. L'étoile symbolise Ishtar, déesse de l'amour, de la procréation et de la guerre qui régit la vie et la mort. $C f$. hbaltzinger@canalblog.com.

5. Dorothy K. Burnham, L'Art des étoffes. Le filage et le tissage traditionnels au Canada, Ottawa, Galerie nationale du Canada. Musées nationaux du Canada. 1981, p. 74.Traduction de The Comfortable Arts : Traditional Spinning and Weaving in Canada.

6. Marius Barbeau, Au cœur du Québec, Montréal, Les Éditions du Zodiaque, 1934, p. 34.

7. Michel Laurent. op.cit. p. 13-16. Les motifs variés et colorés des couvertures boutonnées traditionnelles de Charlevoix sont d'ordre zoomorphique et anthropomorphique, phytomorphique, cosmographique et géomorphique.

8. Ibid. p. 10. 


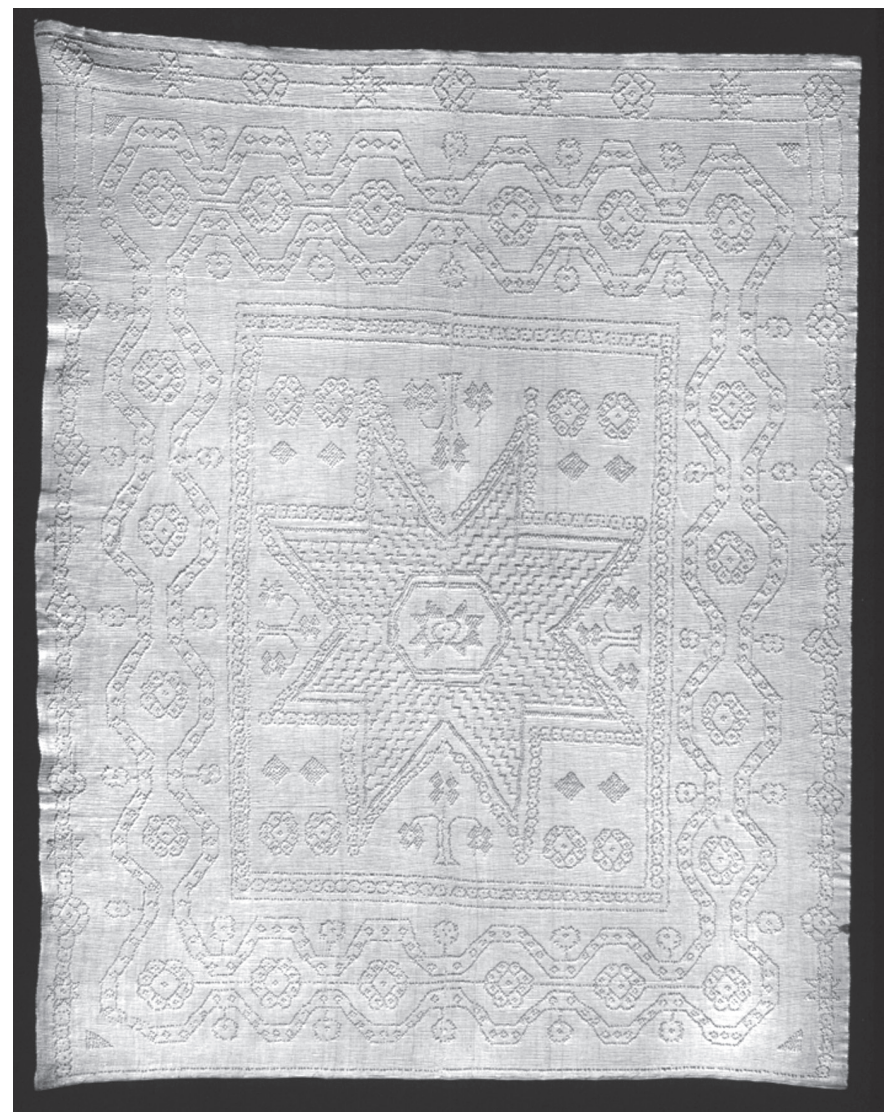

Couvre-lit blanc tissé en deux laizes réunies à la couture-main, vers 1860 Armure toile avec dessins au point boutonné.

Inspiré des modèles britannique et anglo-américain du XIX ${ }^{\mathrm{e}}$ siècle.

Source : Musée royal de l'Ontario, Toronto, Ontario.

Don de $\mathrm{M}^{\mathrm{me}}$ John David Eaton (970.90.6)

Véritable chef-d'œuvre tissé par Alida Thiboutot de Sainte-Anne-de-la-Pocatière, Québec, avec le lin qu'elle a cultivé et filé elle-même. L'œuvre lui méritera un premier prix à l'exposition agricole provinciale.

de la vie quotidienne malgré des difficultés d'approvisionnement en coton notamment. Pour Barbeau, cette découverte correspond à l'idéologie de sauvegarde de tout objet-témoin du génie canadien-français issu du milieu rural et explique en partie l'urgence de sa collecte. Ce regard l'emporte en général sur des considérations comme le contexte de production, le savoirfaire, l'outil de travail, la matière, le mode de transmission, voire aussi l'artisan qui a créé l'œuvre. Plus largement, l'intérêt pour ce tissage particulier répond 
à la philosophie du mouvement occidental Arts \& Crafts alors en vogue, qui favorise l'artisan et l'artisanat par opposition au produit industriel' ${ }^{9}$.

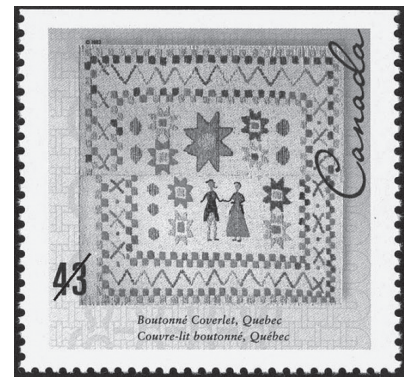

\section{Timbre-poste canadien émis le 30 avril 1993 pour souligner l'année de l'artisanat des Amériques}

Parmi les cinq timbres évoquant la tradition textile du Canada,

le Québec est représenté par le couvre-lit boutonné de Charlevoix. L'œuvre, aux couleurs vives, aurait été tissée vers 1893 par Angèle Perron du rang

Pie-Sec à Saint Urbain. Réalisé en deux laizes et réuni à la couture-main, l'ouvrage comporte également une deuxième technique traditionnelle, le tissage à la planche.

Outre sa mission de collecte de couvre-lits pour les musées canadiens et des collectionneurs privés, Barbeau fait connaître le boutonné à travers ses publications et conférences publiques, se fait animateur de soirées culturelles avec démonstration de la technique et organisateur ou participant d'expositions au Québec comme à l'étranger ${ }^{10}$. À ce chapitre, il faut lui reconnaître son ouverture sur l'art alors qu'il n'hésite aucunement à exposer sous le même toit les œuvres des peintres canadiens du Groupe des Sept et l'artisanat textile du Québec comme le boutonné ${ }^{11}$. Selon Benoît Thériault du Musée canadien de l'histoire, «Barbeau se fait le promoteur des arts textiles traditionnels à qui veut l'entendre. Bien évidemment auprès de son entourage

9. Linda Parry, William Morris and the Arts and Crafts Movement. A Design Source Book, London (England), Studio Editions, 1989, 100 pl. coul. : Linda Parry. « Introduction », p. 6-15 ; Gillian Moss, " Essay on Textiles of the American Arts and Crafts Movement », p. 16-22. Ces deux textes montrent comment, à ses origines vers 1880 , le mouvement visait l'intégration du savoir-faire de l'artisan, du travail en atelier et de la manufacture, et combien, avec le temps, cet idéal fut dilué face au rouleau compresseur du travail à la chaîne et des exigences du marché.

10. En 1937, en prévision de l'exposition universelle à Paris, trois couvertures boutonnées du Québec figurent parmi les objets d'artisanat sélectionnés par la Canadian Handicrafts Guild de Montréal. Barbeau y participe en prêtant des couvre-lits. Deux des couvertures boutonnées proviennent de l'île aux Coudres dont celle qui figurera sur le timbre-poste 1993, œuvre d'Angèle Perron du rang Pie-Sec à Saint-Urbain. La deuxième est de facture anonyme et la dernière, monochrome et d'inspiration « angloaméricaine » est d'Alida Thiboutot de Sainte-Anne-de la Pocatière .

11. Entre 1926 et 1947 , le boutonné figure dans une vingtaine d'expositions et soirées culturelles organisées par Barbeau. À compter de 1947, Jean-Marie Gauvreau prendra le relais des démonstrations de savoir-faire. 
et de ses connaissances, mais aussi auprès d'institutions privées comme le Château Frontenac à Québec et le Château Laurier à Ottawa, qu'il convainc de créer des pièces ou chambres typiquement "canadiennes"12 ».

Dans l'intervalle, la notion de patrimoine culturel évolue néanmoins grâce à des initiatives d'acteurs comme Jean-Marie Gauvreau (1903-1963) et Paul Gouin (1898-1976), pourtant situés en marge du milieu de la recherche. Tout comme Barbeau, les deux hommes s'intéressent vivement à la sauvegarde du patrimoine mais à partir de visées autres. C'est-à-dire audelà de la collecte et de la conservation. Par exemple, au cours des années 1930-1944, Gauvreau, alors directeur de l'École du meuble à Montréal, fait œuvre de pionnier en proposant de concilier les notions de patrimoine, d'économie, de développement touristique et de territoires. Ce qui constitue une vision très d'avant-garde pour l'époque ${ }^{13}$. Bernard Genest, qui le décrit comme un pragmatique ${ }^{14}$, analyse son parcours en concluant qu'il « est sans doute l'un des premiers à avoir compris que tourisme et culture font partie d'une même industrie et que l'économie des régions repose en partie sur la mise en valeur de ses terroirs ${ }^{15}$. »

Son contemporain Paul Gouin, qui a quitté la politique active pour prêcher " l'intégration de l'héritage culturel dans la vie quotidienne ${ }^{16}$ " partage la vision holistique du patrimoine avancée par Gauvreau et son intérêt pour les artisans ${ }^{17}$. Gouin favorise quant à lui l'intégration des traditions artistiques, non pas dans une visée traditionaliste, mais comme source d'inspiration pour les créateurs et les artisans. Fortement engagé dans la relance de l'industrie touristique, de la mise en valeur des régions et de leurs ressources diverses, il aurait inspiré, dit-on, plusieurs initiatives gouvernementales en ce sens ${ }^{18}$. La vision de ces deux missionnaires du patrimoine, soutenue par l'immense inventaire détaillé (1937-1944) que Gauvreau mène à travers les régions du Québec, participe à faire évoluer la notion de patrimoine et à considérer que des biens culturels peuvent être plus que des monuments isolés ou des artefacts à conserver. Bref, que le patrimoine peut être vivant. Cependant, il reste encore du chemin à parcourir pour que l'idée s'implante et rejoigne le milieu universitaire.

12. Benoit Thériault, correspondance, 24 mars 2014.

13. Bernard Genest, «Ethnologie des terroirs », Entre Beauce et Acadie. Facettes d'un parcours ethnologique. Textes réunis par Jean-Pierre Pichette avec la collaboration de Jocelyne Mathieu, Richard Dubé et Yves Bergeron, Québec, Presses de l'Université Laval, 2001, p. 155-177, p. 164.

14 Ibid., p. 161.

15. Ibid. p. 164.

16. Extraits du compte rendu de Bernard Genest de la publication de Nathalie Hamel, « Notre maître le passé, notre maître l'avenir ». Paul Gouin et la conservation de l'héritage culturel du Québec, Québec, Les éditions de l'IQRC, 2008, 204 p., Rabaska, vol. 7, 2009, p. 200.

17. Ibid., p. 200-201.

18. Ibid., p. 202. 
Durant la même période en 1944, Luc Lacourcière, disciple de Barbeau, crée les Archives de folklore de l'Université Laval. C'est sans bruit et en marge de la recherche sur la tradition orale que la culture matérielle fait son entrée à l'université, grâce aux recherches sur le costume régional du Québec réalisées par Madeleine Doyon-Ferland et des cours d'été donnés par Marius Barbeau. On note qu'en 1960 le cours de méthodologie de Luc Lacourcière comprenait une initiation à la culture matérielle ${ }^{19}$. L'incursion dans ce domaine suscitera l'intérêt de l'élève Jean-Claude Dupont qui ouvrira grand les vannes de ce champ d'étude où tout était à faire au Québec. Impossible ici de nommer tous les chercheurs et étudiants qui ont poursuivi dans cette voie au cours des années 1970 et 1980, motivés, il faut le reconnaître, par la montée du nationalisme québécois et l'éclatement de la société traditionnelle. C'est dans ce contexte, joint à un mouvement de retour à la terre du citadin à la recherche de ses « racines », que l'étude de la culture matérielle prend son envol et que l'intérêt pour le boutonné se manifeste. En témoignent des inventaires et des publications sur l'artisanat québécois, les intérieurs domestiques et des pratiques traditionnelles, de même que la recherche sur des artisans et leurs savoir-faire. Actuellement, des techniques comme le boutonné profitent d'outils modernes de diffusion comme l'exposition interactive du studio Trad du Centre culturel Paul-Médéric à Baie-Saint-Paul et le portail électronique IrEPI à l'Université Laval (Inventaire des ressources ethnologiques du patrimoine immatériel).

Dans les annales de la reconnaissance du patrimoine culturel on ne peut passer sous silence l'année phare 2002 où l'UNESCO définit enfin les paramètres des biens culturels. «Pas moins de vingt catégories de biens étaient reconnues pour illustrer la diversité du concept, dont l'artisanat qui renvoie à des savoirfaire ». "Voilà donc, comme le souligne Louise Brunelle-Lavoie, alors présidente de la Commission des biens culturels du Québec, le patrimoine immatériel reconnu au même titre que les traces matérielles laissées au cours de l'histoire ${ }^{20} »$.

Sur le terrain, dans Charlevoix, les communautés de vie n'ont pas attendu les déclarations officielles, les études ou les inventaires pour réaliser et faire connaître le boutonné ou pour faire évoluer la technique. Rappelons que depuis le milieu du $\mathrm{XIX}^{\mathrm{e}}$ siècle la région est déjà reconnue comme une destination touristique prisée par une clientèle nantie et que son artisanat textile est recherché pour ses couvertes de laine tissées et ses tapis crochetés

19. «L'Étal discret du sage », Entrevue avec Jean-Claude Dupont. Propos recueillis par Jean-Pierre Pichette, «Entre Beauce et Acadie. Facettes d'un parcours ethnologique, op. cit., p. 22.

20. Louise Brunelle-Lavoie, « Patrimoine : une notion qui évolue », Cyril Simard, Des métiers... de la tradition à la création. Anthologie en faveur d'un patrimoine qui gagne sa vie, Québec, Les Éditions GID, Tome 1, 2003, p. 52. 
notamment ${ }^{21}$. Bien avant la découverte « officielle » du boutonné par Marius Barbeau en 1925, l'industrie domestique et locale tissait une couverte en laine fine destinée à l'estivant et au touriste de croisière ${ }^{22}$. Cette couverture simple, rapide et économique à réaliser, portait le nom savoureux de « baiseuse $»^{23}$. Lorsque le boutonné, sanctionné de surcroît par un scientifique, devint populaire, on l'ajouta à la production des couvertures tissées avec la différence que le temps investi en faisait un objet de luxe. Pour la région donc, la découverte du boutonné de Charlevoix constitue une valeur ajoutée aux attraits et produits touristiques existants. L'industrie locale ira jusqu'à en breveter certains modèles. Voilà toute une transformation pour un objet du quotidien, à l'origine créé en situation d'autarcie.

La mise au grand jour du boutonné traditionnel en 1925 mène cependant à une importante mutation au niveau de la technique de fabrication et de son esthétique. De fait, en moins de cinq ans, le savoir-faire fait l'objet d'une petite révolution venue de la rive sud du Saint-Laurent, un peu à l'image des contes, chansons et légendes qui se métamorphosent avec le passage du temps et selon le «portageur ». Dans la situation qui nous intéresse, l'instrument du changement est Émilie Chamard, tisserande de SaintJean-Port-Joli. Euvrant au ministère de l'Agriculture de 1928 à 1930 puis comme technicienne-enseignante à l'École des arts domestiques de 1930 à 1946, elle analyse le boutonné pour en simplifier la réalisation. Recourant d'abord à des outils communs comme le crochet pour relever les bouclettes - en remplacement des doigts - et des broches à tricoter et des pailles pour les maintenir en place, elle poursuit ensuite en créant de nouveaux motifs, tôt diffusés dans tout le Québec via la revue des Cercles de fermières et les publications d'Oscar Bériau alors directeur de l'École des arts domestiques ${ }^{24}$. Sauf de rares exceptions, la majorité des tisserandes adoptent d'emblée la méthode Chamard. La Malbaie est d'ailleurs une des premières localités en 1928 à faire venir la tisserande pour enseigner la nouvelle façon de faire et proposer des patrons et motifs prêts à copier. Du boutonné traditionnel de Charlevoix, madame Chamard retient l'apport en couleur, la laine pour

21. François Tremblay, «Artisans du Québec », Cyril Simard, op.cit., p. 72-87.

22. Ibid., p. 78. À titre d'exemple, en 1915, Alcide Bergeron ouvre la boutique Little Shop à Pointe-au-Pic, qui devint le plus important centre de production et de distribution d'artisanat de la région.

23. Ibid., p. 81. Parmi les cinq commerces qui vendaient la «baiseuse » au cours des années 1930, certains en écoulaient entre 5000 et 6000 par année, exportation comprise. Les ouvrières touchaient entre $0,40 \$$ et $0,50 \$$ par couverture et une bonne ouvrière pouvait en tisser entre six et huit dans une journée. La couverture boutonnée, plus grande et plus longue à produire, rapportait $1 \$$ plus $0,25 \$$ pour la teinture.

24. On se rappelle que la majorité des publications d'O. Bériau relatives au tissage ont été traduites en anglais et ont connu une large diffusion au Canada et aux États-Unis. Lire à ce sujet Anne-Marie Poulin, « La Tradition textile au Québec : une tradition du geste, de la parole et... de l'écrit », Rabaska, vol. 12, 2014, p. 153-159. 
réaliser les bouclettes et, au niveau des motifs, l'étoile dite de Charlevoix. En dépit de cette (r)évolution modifiant le geste traditionnel du faire et la créativité, l'appellation d'origine - boutonné de Charlevoix - déjà gravée dans la mémoire collective, demeure intacte.

Depuis ce temps, la transmission de la technique repose presque exclusivement sur les membres des Cercles de fermières et, jusqu'en 1988, sur les techniciennes du ministère de l'Agriculture. De fait, à compter des années 1930 et de façon cyclique, le boutonné façon Chamard (épuré et standardisé) refait surface et fait l'objet de cours, de concours, d'ateliers, d'expositions et de publications modestes, assurant ainsi la pérennité de la technique entre les membres. L'intérêt pour le boutonné s'étend également à des guildes et groupes d'études en tissage au Québec et à l'étranger. En témoigne le congrès international des tisserands « Convergences » à Toronto en 1986 où le boutonné est à l'honneur et où deux représentantes de la maison Routhier à Québec sont sollicitées : l'une pour donner des ateliers en français et en anglais, l'autre pour tisser 400 échantillons de boutonné à remettre aux participants ${ }^{25}$.

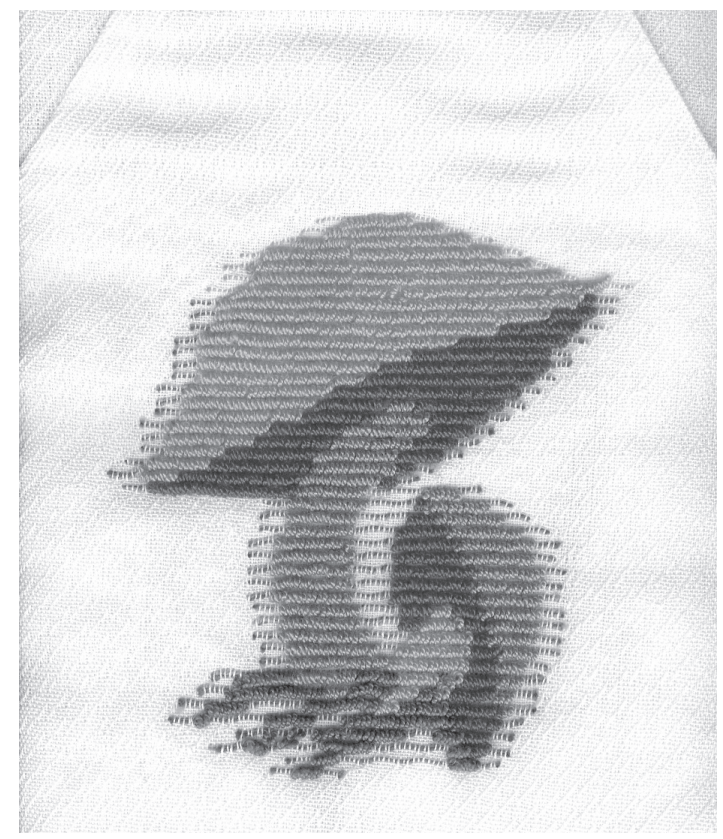

Boutonné contemporain

Création Antoinette Roy, Québec, 1983.

Titre : fantaisie de mycologue, 8 cadres, métier basse-lisse.

Base en coton. Bouclettes en coton lustré et acrylique à crocheter.

25. Monique Dumas et Antoinette Roy. 
En 1'an 2000, à Québec, l'UNESCO tient une rencontre internationale de trois jours sur le thème " Tissage et métissage » avec démonstration de techniques traditionnelles de tissage dont le boutonné. Cette année, en 2015, l'Association des tisserands du Québec inclut un atelier sur le boutonné lors de son congrès annuel avec, comme suivi, un concours en 2016. S'il est vrai que la technique découverte par Barbeau s'adresse davantage aux initiés et a tendance à évoluer en vase clos, l'objet traditionnel pouvant servir de modèle semble inaccessible. D'où l'intérêt d'exposer et de faire ciculer les collections de couvertures depuis trop longtemps rangées dans les musées, pour les valoriser auprès du grand public et stimuler la créativité parmi les artisanes. Sauf erreur, la dernière exposition où le boutonné trouvait place eut lieu à la Galerie nationale du Canada à Ottawa, aujourd'hui Musée des beaux-arts du Canada, en $1981^{26}$.

Actuellement, dans Charlevoix, le tissage d'articles divers en boutonné s'inspire de plusieurs sources, tant traditionnelles que modernes, intégrant même des matières venues d'ailleurs comme l'alpaga. À des fins commerciales toutefois, on relève moins de dix tisserandes du boutonné dans la région. L'hôtel La Ferme à Baie-Saint-Paul, qui encourage la mise en valeur des talents et des produits régionaux, mandatera une de ces tisserandes pour réaliser des objets décoratifs pour ses besoins ${ }^{27}$.

Il y a tant à faire dans le domaine de la tradition textile au Québec, et le boutonné, découvert par Marius Barbeau, constitue un point de départ des plus fertiles. Bien que la pertinence de la découverte semble probante, il y a lieu d'approfondir la recherche sur le rayonnement dont elle a été l'objet de 1925 à 1980, sur les modes de diffusion empruntés par Marius Barbeau pour faire connaître le produit et son procédé, sur la diversité des motifs et leur symbolisme, sur les divers contextes de production d'hier et d'aujourd'hui, sur le parcours des artisans travaillant dans l'ombre et l'évolution de leur savoir-faire ${ }^{28}$ de même que des matières utilisées. Enfin, ce champ d'étude ne saurait être complet sans définir ces régionalismes avec leurs variantes ou dérivés liés à la literie tissée dans Charlevoix, tels le boutonné/boutonnu et le paresse-boutonné/paresse-boutonnu, la catalogne de lit de même que la baiseuse et le bâtard, la « couverture de la mariée » que l'on retrouve aussi

26. Catalogue d'exposition : Dorothy K. Burnham, L'Art des étoffes. Le tisssage et le filage traditionnels au Canada, Ottawa, Galerie Nationale du Canada, Musées nationaux du Canada, 1981, 238 p., diagr. et planches.

27. Anne-Marie Hamel, de Clermont, est mandatée pour réaliser le projet. Voir aussi le portail de l'hôtel La Ferme : www.lemassif.com.

28. Bernard Genest, op.cit. p. 176. Notant que la problématique du savoir-faire est encore très peu étudiée, Genest rappelle que le terme peut comporter deux sens : « le savoir-faire codifié (la recette) [...] et le savoir-faire non codifié qui résulte d'expériences particulières, dont le mode de transmission demeure essentiellement oral et visuel ». Sur cette problématique, $c f$. Denis Chevallier, « Des savoirs efficaces », Terrain 16, Paris, mars 1991. p. 7. 
bien dans la région charlevoisienne qu'en Acadie et au Québec de même que la couverture de La Malbaie qui semble être, selon certains, un générique pour la production régionale. Autant de termes et expressions qui n'ont pas encore trouvé place dans des dictionnaires québécois ou de parler populaire ${ }^{29}$. Enfin, nous savons que Michel Laurent, ethnologue, conservateur-retraité des Musées de l'Amérique française et collectionneur-spécialiste du textile dont le boutonné, poursuit sa recherche sur les textiles anciens du Québec. Cette recherche viendrait compléter l'œuvre amorcée par Barbeau il y a un siècle ${ }^{30}$. Vivement une monographie sur le boutonné !

29. Une revue rapide d'une quinzaine d'ouvrages montre que, seul, le mot boutonné trouve place dans une publication : Gaston Dulong, Le Dictionnaire des canadianismes. Nouvelle édition revue et augmentée, Québec, Septentrion, 1999, 550 p. Le terme est classé uniquement comme adjectif, p. 75.

30. Michel Laurent, "Lire entre les trames, étude d'une production familiale boutonnée à l'Île-aux-Coudres ", Continuité, no 134, automne 2012 p. 16-17. Texte disponible sur http://www. youscribe.com/catalogue/tous/continuite-no-134-automne-2012-2511385. Il a également prononcé une conférence sur l'histoire du boutonné à Clayton, état de New-York, en 2014. 